\title{
Design of Compensated Thermal Neutron Detector Based on He-3 Tube of SiC Micro-structure
}

\author{
Jianlu $\mathrm{Wu}^{1}$, Hui Guo ${ }^{1}$, Xiaoyan Tang $^{1}$, Yimen Zhang ${ }^{1}$, Yimeng Zhang ${ }^{1}$, Yuming \\ Zhang $^{1}$
}

1.School of Microelectronics, Xidian University, Xi'an, 710071, China

Email: wjl6213224@qq.com

\begin{abstract}
Keywords: silicon carbide; micro-structure; He-3 tube; thermal neutron; detector
\end{abstract}
\begin{abstract}
A design scheme for compensated thermal neutron detector based on He-3 tube of SiC micro-structure is proposed herein in order to improve the detection sensitivity of the thermal neutron detector and then to improve the detection precision. Firstly the physical foundation analysis of thermal neutron detector with the silicon carbide (SiC) micro-structure is given herein and it is pointed out that the instrument response basically reflects the tiny space of stratum full of fluid; secondly, the response equation of He-3 Tube structure of the neutron detector is given and then the functional block diagram for compensated neutron detector is designed based on it; finally, the performance of compensated thermal neutron detector based on He-3 tube of SiC micro-structure proposed herein is effectively tested through experiment.
\end{abstract}

\section{Introduction}

Since the 21st century, with the vigorous development of semiconductor industry, the rapid advance of growth process of semiconductor material, devices fabrication process and the technology of large scale integrated circuit, as well as the continuous decrease in production and processing cost have laid an important foundation for the profound research on the semiconductor neutron detector based on micro-structure. Planar semiconductor neutron detector consists of semiconductor diode and coating for conversion of surface neutron, and due to the problems about self-absorption of applied conversion layer, the detection efficiency of the planar semiconductor neutron detector is relatively low (detection efficiency for the thermal neutron less than 5\%). Muminov et al had firstly put forward the concept of semiconductor neutron detector based on micro-structure in 1987 and hence opened up a new field where the micro-structure semiconductor is applied to the neutron detection.

Compared with gas detector, semiconductor is obviously small in volume and the detector made from it can be used for the neutron intensity measurement in narrow space area, and its detection efficiency of thermal neutron can exceed $20 \%$. In case the method of multi-layer stacking is utilized, its detection efficiency of thermal neutron can reach above $50 \%$. Thanks to these advantages of semiconductor neutron detector based on micro-structure, it plays an important role in the measurement of neutron flux density in weak neutron radiation field, the neutron radiation detection on plutonium (uranium) material and spent fuel, the neutron individual dosimetry and neutron scattering measurement and such fields.

For this purpose, a design methods for compensated thermal neutron detector based on He-3 tube of silicon carbide (SiC) micro-structure is proposed herein. The physical foundation analysis of thermal neutron detector with the $\mathrm{SiC}$ micro-structure, operating principle of compensated neutron instrument and response equation of He-3 tube structure of neutron detector are given herein, too, and finally the performance of compensated thermal neutron detector based on He-3 tube of SiC micro-structure is effectively tested through experiment.

\section{Structural design of detector}

Physical foundation for thermal neutron detector based on SiC micro-structure 
The compensated neutron detector adopts $\mathrm{SiC}$ micro-structure and is provided with 20 Curie Am-Be neutron source with the energy of about several million electron volts. It generates $4 \times 107$ fast neutrons per second which inject into the stratum and have a series of nuclear reaction with substance in the stratum. These include: inelastic scattering of fast neutron, excitation of fast neutron to the nucleus and the elastic scattering and deceleration of fast neutron. The energy of the fast neutron gradually decrease due to a series of inelastic collision and elastic collision, and finally when the neutron energy is under the same thermal equilibrium state as the atom in the stratum, the neutron will not decelerate any more. The neutron under this energy state is called thermal neutron. The energy of standard thermal neutron is: $0.025 \mathrm{ev}$ with a speed of $2.2 \times 105 \mathrm{~cm} / \mathrm{s}$. According to the collision theory, the energy loss in neutron in collision is related to the mass and angle of incidence of the substance to be collided, and if the neutron collides with the substance with the mass equivalent to it (elastic collision), the energy loss in neutron is the highest. In the stratum, the hydrogen atom is of the mass very close to the neutron, and therefore the deceleration capacity of the fast neutron in the stratum mainly depends on the hydrogen content of the stratum, and the stronger the macroscopic deceleration capacity of the stratum, the small the deceleration length. Upon several collisions, the fast neutron will be decelerated and the energy from the average one of $5.6 \mathrm{MeV}$ of the fast neutron reduces to $0.025 \mathrm{eV}$ of the thermal neutron. The stratum pore is a ting space full of fluid, and the water and hydrocarbon contain hydrogen atom, and the oil-free stratum and ore-rock barely contains hydrogen. Therefore, the instrument response basically reflects the tiny space of stratum full of fluid, namely the porosity.

He-3 tube structure of neutron detector

Currently, domestic and overseas compensated neutron logging instruments adopt He-3 proportional counter tube as the thermal neutron detector which outputs negative polar electric impulse under normal operating condition, and the impulse amplitude is continuously distributed within the range of $0.5 \mathrm{uv}--1.5 \mathrm{mv}$; if the impulse width is less than $5 \mathrm{us}$, it is a random signal, and the outline structure of He-3 tube is cylindrical and there is theatrically infinitesimal metal wire on the axis of the cylinder in which is full of many He-3 gases with atmospheric pressure, and the range of atmospheric pressure is related to the sensitivity. The response equation for its neutron detection is as follows:

$$
{ }_{2} \mathrm{He}^{3}+{ }_{0} \mathbf{n}^{1} \rightarrow \mathrm{T}+\mathbf{P}+\mathbf{0 . 7 6 4 M e v}
$$

The characteristics of neutron He-3 tube detector are: (1) simple structure; (2) resist high-voltage electricity impact, but not the physical stress impact; (3) infinite load; (4) weak output signal required to be shielded carefully; (5) only sensitive to thermal neutron, but not the neutron of other energies.

Operating principles of compensated neutron instrument

These thermal neutrons partly enter the detector and collide with the He-3 nuclear to induce nuclear reaction and generate $\mathrm{H} 3$ (tritium) proton which makes part He-3 ionization to generate electriferous ion and electron, and under the effect of high voltage electric field, the electron moves to the anode to generate a negative impulse which is magnified and recorded by the electronic circuit, and the quantity of the neutrons received by the detector directly reflects the quantity of the hydrogen atom in the stratum. Therefore, the downhole cartridge consisting of He-3 detector and its electronic circuit can be used to measure the hydrogen content in the stratum. The operating principle block diagram for compensated neutron instrument designed is shown in Fig. 1. 


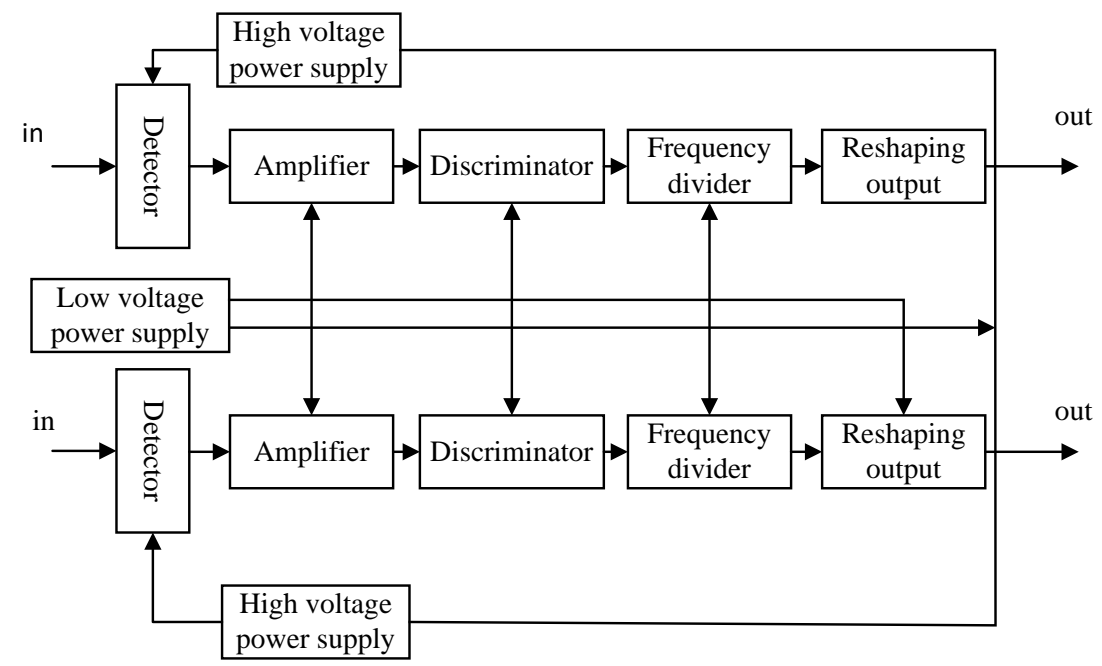

Fig. 1 Block Diagram for Operating Principles of Compensated Neutron Instrument

Suppressing methods for circuit disturbance of compensated neutron instrument include: (1) short circuit between circuit ground wire and the instrument framework. (2) Amplifier shielding case grounding. (3) Connect instrument and the earth with long conductor, which has significant effect on the unknown disturbance, especially in the process of instrument heating. (4) Thin film alloy sheet is used as the shielding case to completely cover part pre-amplifying circuit so as to suppress most disturbances. (5) Cleaning the entrance welding points of the instrument, detector and high voltage amplifier can suppress the persistent disturbance. Capacitance is added between circuit ground wire and the instrument framework, which can suppress the disturbance in instrument logging. The capacity is between 1 microfarad to 4.7 microfarads.

\section{Performance test}

The thermal neutron detector is remarkably characterized by the high intrinsic detection efficiency of the thermal neutron, compared with the traditional semiconductor neutron detector. Table 1 compares the performance of the thermal neutron detector herein and that of the traditional planar detector when the groove clearance and width are $5^{\mu \mathrm{m}}$ and $15^{\mu \mathrm{m}}$ respectively and the depth is $200^{\mu \mathrm{m}}$ (the area of the sensitive region is $1 \mathrm{~cm} 2$ ). The detection efficiency in Table 1 refers to the detection efficiency of thermal neutron when the system discrimination threshold is $300 \mathrm{keV}$. Table 1 shows that the existence of three-dimensional structure greatly enlarges the area of contact between the semiconductor material and neutron conversion material of the thermal neutron detector herein, and meanwhile greatly enlarges the volume of neutron conversion materials for filling. Therefore, the intrinsic detection efficiency of thermal neutron detector herein is essentially improved (compared with the planar detector, its detection efficiency is improved 9.2 times).

Table 1 Effect of Structure on Detection Efficiency

\begin{tabular}{cccc}
\hline detector & $\begin{array}{c}\text { contact area } \\
/ \mathrm{cm}^{2}\end{array}$ & $\begin{array}{c}{ }^{6} \mathrm{LiF} \text { volume } \\
/ \mathrm{cm}^{3}\end{array}$ & $\begin{array}{c}\text { detection } \\
\text { efficiency } / \%\end{array}$ \\
\hline MSND & 20.71 & 0.0150 & 37.77 \\
planar detector & 1.0 & 0.0030 & 4.09 \\
\hline
\end{tabular}

The thermal neutron detector herein belongs to semiconductor detector and inherits the advantage that the traditional semiconductor detector neutron is strong in gamma ray discrimination. Neutron to gamma ray rejection ratio is defined as follows:

$$
\delta_{n / \gamma}=\frac{\varepsilon_{n}\left(E_{n}\right)}{\varepsilon_{\gamma}\left(E_{\gamma}\right)}
$$

Where, $\varepsilon_{n}\left(E_{n}\right)$ and $\varepsilon_{\gamma}\left(E_{\gamma}\right)$ are detection efficiencies respectively for the neutron with energies of $E_{n}$ and $E_{\gamma}$ and the $\gamma$ ray for the detector. $\delta_{n / \gamma}$ value is also related to the system discrimination threshold setting. Method of Monte Carlo simulation is adopted to obtain the 
detection efficiencies of two types of detectors for $137 \mathrm{Cs}$ and $662 \mathrm{keV}$ gamma rays in Table 1 , of which the interaction of photon with materials is described with the Penelope physical model. The total thicknesses of silicon slices of two types of detectors are $0.5 \mathrm{~mm}$. When the discrimination threshold is $300 \mathrm{keV}$, the $\delta_{n / r}$ of the thermal neutron detector herein for the thermal neutron and $662 \mathrm{keV}$ gamma ray of $137 \mathrm{Cs}$ source is up to $4.1 \times 10^{3}, 23.7$ times more than that of the planar detector. Change curve of $\delta_{n / \gamma}$ with the discrimination threshold is shown in Fig. 2.

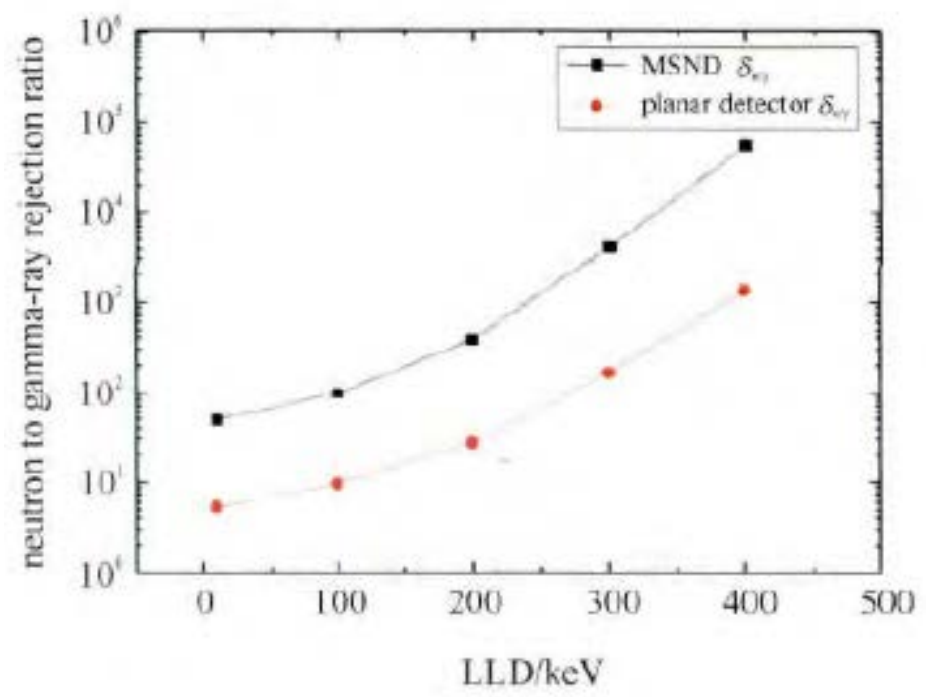

Fig. 2 Change Curve of Neutron to Gamma Ray Rejection Ratio

Fig.2 shows, when the values of discrimination threshold are same, the $\delta_{n / r}$ value of the thermal neutron detector herein is higher than that of $\delta_{n / \gamma}$ of traditional planar detector, and the larger the discrimination threshold, the faster the increase speed of the $\delta_{n / r}$ value of the thermal neutron detector herein than the traditional planar detector. The experiment results verify the effectiveness of proposed thermal neutron detector.

\section{Conclusion}

A design scheme for compensated thermal neutron detector based on He-3 tube of SiC micro-structure is proposed herein and the theoretical foundation for the thermal neutron detector with silicon carbide ( $\mathrm{SiC}$ ) micro-structure is analyzed, the response equation of He-3 tube structure of neutron detector is given based on it and the block diagram for principle design of compensated neutron instrument is given herein, too, and then it is verified through experiment. Sand algorithm is simply verified through experiment during the period of design, and future research will focus on how to apply it to the actual detection more effectively.

\section{Reference}

[1] Zakharov L E, Gorelenkov N N, White R B, et al. Ignited spherical tokamaks and plasma regimes with LiWalls[J]. Fusion Engineering \& Design, 2004, 72(1-3):149-168.

[2] Bresnehan M S, Hollander M J, Wetherington M, et al. Prospects of direct growth boron nitride films as substrates for graphene electronics[J]. Journal of Materials Research, 2013, 29(3):459-471.

[3] Blaich T, Elze T W, Emling H, et al. A large area detector for high-energy neutrons[J]. Nuclear Instruments \& Methods in Physics Research, 1992, 314(1):136-154.

[4] Barrett K, Bragg-Sitton S. Advanced LWR Nuclear Fuel Cladding System Development Trade-Off Study[J]. Office of Scientific \& Technical Information Technical Reports, 2012, 6(37):76. 
[5] Yang, J., Wang, H., Lv, Z., Wei, W., Song, H., Erol-Kantarci, M., ... \& He, S. (2016). Multimedia recommendation and transmission system based on cloud platform. Future Generation Computer Systems. 